\title{
ON THE SPECTRAL CLASSIFICATION OF COOL WHITE DWARFS
}

\author{
I. BUES
}

\author{
I. Mathematisches Institut der Freien Universität Berlin
}

\begin{abstract}
The Eggen-Greenstein (1965) classification of cool white dwarfs (DC, DF, DG, $\lambda$ 4670, $\lambda$ 4135) was based on the features visible in the spectra of low dispersion. Detailed model atmosphere computations $\left(11000 \geqslant T_{\text {eff }} \geqslant 7000 \mathrm{~K}\right)$ and comparison with spectra observed by Wegner (1974, 1975) show that the variety of the spectra is due to changes in the abundance ratios of $\mathrm{C} / \mathrm{O}, \mathrm{C} / \mathrm{H}$ and $\mathrm{H} / \mathrm{He}$ only. Thus the relative strengths of the $\mathrm{C}_{2}$ and $\mathrm{CH}$-bands can be used for classification and guess of $T_{\text {eff }}$ in the range of $T_{\text {eff }} \geqslant 6000 \mathrm{~K}$.
\end{abstract}

According to the characteristics of white dwarf spectral types reported by Greenstein (1960) the white dwarfs were classified by comparison of the strongest features with MK spectral type criteria for the main sequence, if possible (DA HI, DO He II, He I, H I, DB He I, DF Ca II, DG Ca II, Fe I, DK Ca I). Stars not fitting in this scheme were named after the strongest features visible in their spectra $\left(\lambda 4135, \lambda 4670, \lambda 4670 p, C_{2}\right)$ or DC, if no lines deeper than $10 \%$ could be detected. If this variety of spectra is due to large differences in abundances it can be determined by model atmospheres only.

For effective temperatures $>12000 \mathrm{~K}$ computations by Strittmatter and Wickramasinghe (1971), Shipman (1972) and Bues (1970) showed that the classification parameter is the ratio of $\mathrm{H} / \mathrm{He}$, being $>100$ for spectral type DA and $<10^{-4}$ for spectral type DB. For cooler stars this ratio cannot be derived directly due to the high excitation potential of $\mathrm{He}$ I lines. From the high pressure necessary for the line profiles of $\mathrm{Ca} \mathrm{II} \mathrm{H}$ and $\mathrm{K}$, Wegner (1972) found agreement with $\mathrm{H} / \mathrm{He} \leqslant 10^{-4}$ for $3 \mathrm{DF}$ and $\mathrm{DG}$ stars but different abundances for the heavy elements. Detailed investigations of ionizationdissociation equilibria of carbon molecules in helium-rich model atmospheres (Bues, 1973) showed that a second parameter, the ratio of $\mathrm{C} / \mathrm{O}$, is necessary for the interpretation of the spectra. Stars belonging to the spectral type $\lambda 4670$ can change this ratio from 3 to 13 . New observations by Wegner $(1974,1975)$ revealed some stars with weak $\mathrm{HI}$ lines and some with weak $\mathrm{CH}$ and $\mathrm{C}_{2}$ bands in the range of DC stars. Our analysis (Bues and Wegner, 1975) of 4 stars yields a ratio of $\mathrm{C} / \mathrm{H}$ ranging from 3 to $\frac{1}{3}$ for a helium-rich composition $\left(\mathrm{H} / \mathrm{He} \leqslant 10^{-3}\right)$. The four parameters, the abundance ratios of $\mathrm{H} / \mathrm{He}, \mathrm{He} / \mathrm{C}, \mathrm{C} / \mathrm{O}$ and $\mathrm{C} / \mathrm{H}$, are responsible for the structure of the atmospheres and the different strengths of the bands. They vary among white dwarfs of the same spectral type but differing $T_{\mathrm{eff}}$, the most likely reason being a gravitational sorting mechanism.

\section{References}

Bues, I.: 1970, Astron. Astrophys. 7, 91. 
Bues, 1.: 1973, Astron. Astrophys. 28, 181.

Bues, I. and Wegner, G.: 1975, Astron. Astrophys. (in press).

Greenstein, J. L.: 1960, Stars and Stellar Systems 6, Univ. Chicago Press.

Shipman, H. L.: 1972, Astrophys. J. 177, 723.

Strittmatter, P. A. and Wickramasinghe, D. T.: 1971, Monthly Notices Roy. Astron. Soc. 152, 47.

Wegner, G.: 1972, Astrophys. J. 172, 451.

Wegner, G.: 1975, Monthly Notices Roy. Astron. Soc. 171, 637.

Wegner, G.: 1975, Monthly Notices Roy. Astron. Soc. 171, 529.

\section{DISCUSSION}

Bell: Do you have an estimate of the $\mathrm{C} / \mathrm{N}$ ratio in the $\lambda \mathbf{4 6 7 0}$ white dwarfs?

Bues: From the absence of the cyanogen bands a ratio of $\mathrm{C} / \mathrm{N}>10$ has to be assumed for $T_{\text {eff }}>$ $8500 \mathrm{~K}$. In the lower temperature range $\mathrm{C} / \mathrm{N}>100$ is necessary.

Bell: Would you comment on the identification of the $\lambda 4135$ feature with bands of the $\mathrm{He}_{2}$ molecule?

Bues: As already pointed out by Bues (1973) the interpretation of the $\lambda 4135$ feature in EG 129 as $\mathrm{He}_{2}$ cannot be established by model atmosphere calculations. In the range of $T_{\text {eff }}$ possible for a reasonable amount of $\mathrm{N}_{\mathrm{He}_{2}}$ in the excited states a $\log \mathrm{gf} \approx 1.0$ would have to be assumed for the visibility of the $5 \delta$ transition. That value is unreasonably large and, in addition, would result in a broad feature from 4100-4200 $\AA$ and not in a sharp line as indicated from the reproduced spectra and polarisation measurements. In the light of the atmospheres with a different carbon abundance which account not only for the $\lambda \mathbf{4 6 7 0}$ stars but also for some of the DC stars, an identification of the peculiar feature with the $C$ II line $\lambda 4267 \AA$ seems to be more likely. Tentative model atmospheres with magnetic field strengths of $10^{6}-10^{8} \mathrm{G}$ show that an abundance ratio of $\mathrm{He} / \mathrm{C} \approx 50$ and $\mathrm{B} \approx 10^{7} \mathrm{G}$ at $T_{\text {eff }}=12000 \mathrm{~K}, \log g=8 \mathrm{can}$ produce a feature at the position of the band and with the observed equivalent width. 